\section{Metastases limited}

SiR - It has recently been reported by Kun et al. ${ }^{1}$ that poly (ADP-ribose) has an important role in carcinogenesis, and that inhibition of the biosynthesis of this polymer from NAD inhibits carcinogenesis ${ }^{2,3}$. Based on the structural analogy with nicotinamide, these authors tested benzamide and found it to inhibit this biosynthesis, and to prevent carcinogenesis in human fibroblasts ${ }^{2}$.

In Padua we have conducted studies of anticancer activity of analogues of nicotinamide, aiming at interfering with NAD activities $^{3}$. One promising compound, 6,6'-dithiodinicotinic acid (carboxypyridine disulphide, CPDS) was selected for further studies, also because of its unusual reactivity properties. CPDS was found to decrease by $75 \%$ the formation of lung metastases in mice bearing Ehrlich ascites carcinoma ${ }^{4}$; later studies showed CPDS to reduce by as much as $84 \%$ the metastatic spread of Lewis lung carcinoma in mice, and to significantly decrease the rate of growth of the primary carcinoma in these mices. Other analogues of nicotinamide tested had similar effects ${ }^{5}$.

The fact that the same type of compound is active against both carcinogenesis and metastasis suggests an intimate link between the two. Preliminary experiments indeed appear to confirm this relationship.

Institute of Pharmacology,

D. R. GRASSETTI

University of Padua,

35100 Padua, Italy

1. Kun, E., Kirsten, E., Mito, G.E., Kurian, P. \& Kumari H.L. Proc. natn. Acad. Sci. U.S.A. 80, 7219.7223 (1983)

2. Kun, E. et al in Twelfth Steenbock-Lilly Symposium(eds Lennon, D.L.F., Stratman, F.W. \& Zahiten, R.N.) 387-399 (Elsevier, New York, 1982).

Grassetti, D.R. Chemtech 666-671 (November, 1973).

4. Grassetti, D.R. Nature 228, 282-283 (1970).

5. Grassetti, D.R. in Current Chemotherapy and Immunotherapy, Proc. I2th Int. Congress Chemotherapy, Florence Vol Il, 1365-1366 (American Society of Microbiology, Washington, D.C., 1981).

\section{Flagella or cilia?}

SIR - Margulis ${ }^{1}$ emphasized the morphological case for separate terms to denote "flagella" of prokaryotes and eukaryotes. But the proposed resurrection of "undulipodia" for those of the latter (and cilia) is objectionable, as it is far from euphonious and a linguistic hybrid (LatinGreek). If a new term is required, I tentatively suggest mastigion (Greek: "little whip", a diminutive of mastix, "whip"') - for one or other group of organelles. By priority of usage, flagellum should presumably be retained for the eukaryotic organelle, and mastigion used for that of prokaryotes. But mastigion is already familiar to protistologists from taxonomic usage (Mastigophora and so on), so less disruption might result from its application to the eukaryotic structure. Retention of the name flagellum in bacteria would also accord with naming their protein constituent "flagellin".
But is a new term required at all? Both eukaryotic "flagella" and "cilia" have a similar basic " $9+2$ "' structure; although "flagella" are usually longer and less numerous than "cilia", usually beat independently rather than in metachronal rhythm, and sometimes possess a paraxial rod. Professor K. Vickerman has pointed out (personal communication) that none of these features constitutes a constant difference: the numerous "flagella" of Trichonympha and Opalina exhibit metachrony, by no means all "flagella" have paraxial rods, and length is not an absolute criterion. There seems therefore no justification for their distinction. An alternative solution to the introduction of a new term might be to restrict "flagellum" to the prokaryotic organelle, and extend "cilium" to all the eukaryotic structures. Although the taxonomic associations of cilium make this unlikely to appeal to protistologists, metazoan cytologists would probably prefer to retain the term "cilium" than to adopt a totally new term; and objections from protistologists could be met by redefining the Ciliophora by their possession of an infraciliature rather than (less universally) cilia $^{3}$. J. R. BAKER Institute of Terrestrial Ecology,

Culture Centre of Algae \& Protozoa,

\section{Cambridge CB3 ODT, UK}

1. Margutis, L. Biosystems 12, 105-108 (1980)

2. Kenneth, J.H. in A Dictionary of Biological Terms 8 th edn

(Oliver \& Boyd, Edinburgh, (1968)

3. L.evine, N.D. et al. J. Protozool. 27, $37-58$ (1980).

\section{Sea cow discovery}

SIR - The recent discovery of " a virtually complete skeleton" of Steller's sea cow on Bering Island (Nature 306, 415; 1983) has potential importance that may be overlooked. Although numerous skeletons (considerably more than six) of Hydrodamalis gigas ( = "Rhytina stelleri") have been displayed over the past century, not one has been complete and only one (a juvenile specimen in Helsinki) is apparently associated; all the rest are composites of isolated bones. Therefore we remain largely ignorant of such details as the limb proportions, number of ribs and vertebrae, and morphology of the hand skeleton (of which not a single scrap has ever been discovered).

The fact is that several fossil sirenians, including some species directly ancestral to $H$. gigas, are better known osteologically than this one which persisted into historic times. It is to be hoped that the new specimen, if truly associated, will not gather dust in a provincial museum, but will be studied by Soviet palaeontologists and will add to our still-faulty knowledge of one of the most bizarre of all mammals.

\section{Department of Anatomy,} DARYL P. DOMNING

\section{College of Medicine,}

Howard University,

Washington, D.C.20059, USA

\section{Meteorite ages}

SIR - Jon Darius (Nature 308, 18; 1984) says the recovery of the Lost City meteorite was the first time that photographs from synchronized cameras were used to predict the place of fall of a meteorite. It was not; the Pribram, Czechoslovakia, fall of 7 April 1959 was photographed as a bright fireball and a search of the predicted area yielded four fragments, of total weight $5.555 \mathrm{~kg}$, of a possible nineteen ${ }^{1}$. Like Lost City and the third meteorite to be recovered by photographic prediction (Innisfree, Alberta), Pribram had the orbit of an Apollo asteroid².

Darius also says that the Lost City meteorite has an age in the range 4.8-5.5 $\times$ $10^{\circ}$ years but this is older than the "age of the Solar System", about $4.55 \times 10^{\circ}$ years, based on isotopic studies in meteorites. The only material claimed to be older occurs as inclusions in the Allende meteorite, two of which have yielded ages of about $4.9 \times 10^{9}$ years. However, the interpretation of the Ar-Ar data that produced these data has been questioned (see references in ref. 3).

ROBERT HUTCHINSON

Department of Mineralogy,

British Museum (Natural History), Cromwell Road, London SW7 SBD, UK

1. Rost, R. Casopis min. geol. 5, 423 (1960)

2. Halliday, I. et al. J. R. Astron. Soc. Can. 72, 15 (1978). 3. Jessberger, E.K. Meteorics 17, 232 (1982)

\section{Unnameable named}

SIR - In the report on the preparation of soluble conducting polymers (Nature 304, 487 ; 1983) mention is made of the "unnameable" precursor polymer III with the following structural repeating unit:<smiles>CC=CC1C2C=CC(C1C(C)(C)C)C2C(F)(F)F</smiles>

In fact, the above polymer should be named following the recommendations of the International Union of Pure and Applied Chemistry' as poly[[5,6-bis(trifluoromethyl)bicyclo[2.2.2] octa-5,7dien-2,3-ylene]vinylene]. The corresponding current Chemical Abstracts index name $^{2}$ is poly[[5,6-bis(trifluoromethyl)bicyclo[2.2.2] octa-5,7-diene-2,3-diyl]-1,2ethenediyl].

\section{International Committee on}

KURT L. LOENING

Nomenclature and Symbols,

International Union of

Pure and Applied Chemistry,

PO Box 3012, Columbus,

Ohio 43210, USA

1. IUPAC, Macromolecular Division. Commission on Macromolecular Nomenclature Pure appl. Chem. 48, 373-385 (1976).

2. Index Guide Section 222. (Chemical Abstracts Service, Columbus, Ohio, 1982). 